\title{
Activité réflexive novatrice auprès d'étudiantes en sciences infirmières sur la diversité culturelle dans les soins
}

Sophie Huard

University of Montreal, shuard16@gmail.com

Amélie Blanchet Garneau

amelie.blanchet.garneau@umontreal.ca

Jacinthe I. Pepin

Université de Montréal, jacinthe.pepin@umontreal.ca

Caroline Larue

University of Montreal, caroline.larue@umontreal.ca

Follow this and additional works at: https://qane-afi.casn.ca/journal

Part of the Nursing Commons

\section{Recommended Citation}

Huard, Sophie; Blanchet Garneau, Amélie; Pepin, Jacinthe I.; and Larue, Caroline (2016) "Activité réflexive novatrice auprès d'étudiantes en sciences infirmières sur la diversité culturelle dans les soins," Quality Advancement in Nursing Education - Avancées en formation infirmière: Vol. 2: Iss. 2, Article 6.

DOI: https://doi.org/10.17483/2368-6669.1069

This Article is brought to you for free and open access by Quality Advancement in Nursing Education - Avancées en formation infirmière. It has been accepted for inclusion in Quality Advancement in Nursing Education - Avancées en formation infirmière by an authorized editor of Quality Advancement in Nursing Education - Avancées en formation infirmière. 


\section{Introduction}

La formation en sciences infirmières doit préparer les futures infirmières à offrir des soins non discriminatoires et à composer avec les différentes réalités entourant les soins en contexte de diversité culturelle (AACN, 2008; Blanchet Garneau, 2013). Par ailleurs, certaines personnes, telles que les personnes de groupes ethniques minoritaires, les populations vivant en contexte de vulnérabilité ou marginalisées sont victimes d'inégalités lorsqu'elles reçoivent des soins (McGibbon, Etowa, et McPherson, 2008; Newbold, 2005; Smedley, Stith, et Nelson, 2003). Ces inégalités se définissent par un écart dans la qualité des soins offerts à différentes personnes par un professionnel de la santé, sans égard à leur situation de santé (Smedley et al., 2003). Par exemple, les résultats de la revue systématique de van Boekel, Brouwers, van Weeghel, et Garretsen (2013) montrent que les attitudes stigmatisantes des professionnels de la santé envers les utilisateurs de drogues nuisent à la collaboration de ces personnes dans leurs soins ainsi qu'à leur sentiment d'empowerment. L'infirmière aurait aussi tendance à limiter ses visites aux personnes envers lesquelles elle a des préjugés et à se centrer davantage sur la tâche que sur la personne (van Boekel et al., 2013).

Afin de préparer les infirmières à intervenir avec une population multiculturelle et de réduire les inégalités dans les soins, des chercheurs (Allen, 2010; Beach et al., 2005; Blanchet Garneau, 2013; Gallagher et Polanin, 2015; Horvat, Horey, Romios, et Kis-Rigo, 2014) ainsi que des associations infirmières (AIIC, 2010; RNAO, 2007) recommandent de mettre en place des stratégies, autant en formation initiale qu'en formation continue, afin de permettre aux infirmières de développer leur compétence culturelle. Selon Blanchet Garneau, la compétence culturelle se définit comme étant

(...) un savoir-agir complexe fondé sur la réflexion critique et l'action par lequel le professionnel de la santé vise à atteindre la capacité d'offrir des soins culturellement sécuritaires, adaptés et efficaces en partenariat avec des personnes, familles et communautés vivant une expérience de santé et en prenant en considération les dimensions sociales et politiques du soin. (Blanchet Garneau, 2013, p.213)

Cette définition est issue à la fois des sciences de l'éducation et de la santé. Elle souligne la nature relationnelle, dynamique, évolutive, développementale de la compétence (Blanchet Garneau et Pepin, 2015a). Elle est ancrée dans une conception constructiviste de l'apprentissage où les savoirs antérieurs et les nouveaux savoirs sont développés de manière dialectique par un processus de réflexion et d'action. Cette définition de la compétence culturelle diffère d'autres définitions plus largement utilisées en sciences infirmières telles que la définition de CampinhaBacote (2007). En effet, la culture est considérée selon cette perspective comme étant un processus relationnel dynamique. Les personnes vivent dans des structures et des processus historiques, sociaux, politiques, économiques, physiques et linguistiques et choisissent de répondre à certains éléments de contexte et à les intégrer ou pas. L'intégration des dimensions sociales et politiques du soin dans la définition de la compétence culturelle permet ainsi de prendre en considération ces structures et processus et de nourrir le processus de réflexion critique et d'action qui est au cœur du développement de cette compétence (Blanchet Garneau et Pepin, 2015a). Cette conceptualisation amène à reconnaitre, comprendre et valoriser les différences lorsqu'elles sont présentes et à éviter de les banaliser. Elle fait ainsi dévier de la différence culturelle comme source de problème, vers la culture des systèmes de santé comme autant d'univers de sens à questionner et à inclure en transformant des pratiques de soins normatives pour répondre aux besoins des populations en marge de la société. 
Malgré la convergence de la position des chercheurs en faveur du développement de la compétence culturelle, il n'y a pas de consensus quant à la stratégie d'enseignement à adopter afin de favoriser l'apprentissage des étudiantes ${ }^{1}$ en sciences infirmières (Horvat et al., 2014; Long, 2012). Effectivement, la manière d'intégrer les activités visant le développement de la compétence culturelle dans un programme varie d'un milieu à l'autre. De plus, différents cadres de référence sont utilisés par les enseignants pour élaborer les objectifs d'apprentissage ou les éléments de compétence visés ainsi que les activités d'enseignement elles-mêmes (Blanchet Garneau, 2013). Le stage, la simulation clinique, le groupe de discussion et le cours magistral sont des exemples d'activités d'enseignement. Cette multitude d'approches et de stratégies crée un défi pour évaluer et comparer les activités d'enseignement entre elles, d'autant que nous possédons peu d'informations sur la définition de la compétence culturelle utilisée (Gallagher et Polanin, 2015).

Ainsi, afin de contribuer au développement des meilleures pratiques en formation infirmière, cet article présente l'élaboration et la mise à l'essai au sein d'une université québécoise d'une activité d'enseignement visant à stimuler la réflexion d'étudiantes au premier cycle en sciences infirmières sur les différentes réalités entourant la pratique en contexte de diversité culturelle.

\section{Cadre de référence}

Le modèle du développement de la compétence culturelle de Blanchet Garneau (2013; Blanchet Garneau et Pepin, 2015b), élaboré à partir d'une recherche qualitative selon une approche de théorisation ancrée et une perspective constructiviste, a servi de cadre de référence. Selon l'auteure, la culture d'une personne est dynamique et évolue dans le temps. Elle n'est pas essentiellement associée à l'ethnicité, mais elle prend en considération les intersections entre diverses positions sociales de l'individu telles que le statut socioéconomique, l'orientation sexuelle, l'état matrimonial, l'âge ou le genre d'une personne. Selon Blanchet Garneau et Pepin (2015a), le développement de la compétence culturelle vise à offrir des soins culturellement sécuritaires, adaptés et efficaces. L'intégration de la sécurité culturelle dans cette définition, comme étant une résultante du développement de la compétence culturelle, permet de comprendre les pouvoirs en cause dans la relation de soin et d'agir en conséquence tant au niveau individuel que systémique afin d'équilibrer les pouvoirs et changer les structures et pratiques de soins pouvant reproduire, par exemple, la discrimination systémique et individuelle. Le développement de la compétence culturelle vise aussi à offrir des soins adaptés puisqu'ils prennent en compte la diversité des contextes sociaux, historiques, politiques et économiques, et efficaces, puisqu'ils répondent aux priorités locales et aux besoins spécifiques des patients, familles et communautés (Blanchet Garneau et Pepin, 2015a).

Ce modèle a été retenu puisque, contrairement aux autres modèles (Campinha-Bacote, 2011; Giger et Davidhizar, 2012; Purnell, 2002), il présente des niveaux de développement de la compétence culturelle. De plus, il a été conçu spécialement pour guider la formation des infirmières ou d'étudiantes en sciences infirmières. Ce modèle (Blanchet Garneau et Pepin, 2015) présente l'évolution de la compétence culturelle en trois niveaux. Selon l'auteure, les étudiantes devraient se situer au premier niveau à la fin de leur formation initiale, c'est-à-dire qu'elles devraient être capables de : «s'ouvrir aux différentes réalités entourant la pratique en contexte de diversité culturelle »(Blanchet Garneau, 2013, p. 181). Comme l'activité

\footnotetext{
${ }^{1}$ Le féminin est employé tout au long du document pour alléger le texte et désigne autant le masculin que le féminin
} 
d'enseignement mise à l'essai dans le cadre de ce projet était prévue pour des étudiantes en sciences infirmières, elle a été élaborée en fonction de ce premier niveau. À ce niveau, pour la dimension Construire la relation avec l'autre, l'étudiante perçoit un écart entre sa culture et celle du patient. Elle démontre de l'ouverture à découvrir celle du patient. Elle prend aussi conscience de l'importance de la communication dans les soins en contexte de diversité culturelle pour mieux comprendre l'autre et pour pouvoir adapter ses interventions à chaque personne. En lien avec la dimension Sortir du cadre habituel de pratique, l'étudiante prend conscience des défis entourant la pratique en contexte de diversité culturelle ainsi que des limites structurelles. Par exemple, l'étudiante se rend compte de ce qu'elle doit faire pour adopter une approche de soins davantage centrée sur le patient et sa culture. En d'autres mots, elle prend conscience des barrières à l'adoption d'une approche sensible à la culture de chaque personne. Pour la dernière dimension, Réinventer sa pratique dans l'action, l'étudiante prend conscience de l'écart entre la théorie et la pratique et elle imite l'approche de collègues plus expérimentées (Blanchet Garneau, 2013). Selon ce modèle, le développement de la compétence culturelle est initié par l'immersion clinique de l'étudiante en contexte de soins. Un processus de réflexion et d'action est nécessaire pour évoluer à travers les trois niveaux (Blanchet Garneau, 2013). La trajectoire personnelle de l'étudiante ainsi que les dimensions sociales et politiques du soin influencent aussi le développement de sa compétence culturelle. Ces dimensions comprennent le contexte entourant la relation entre l'étudiante et le patient, dont la structure organisationnelle du milieu de soins ainsi que les valeurs de la société dans laquelle s'inscrit la relation de soins (Blanchet Garneau, 2013). Une recension des écrits a été réalisée afin d'explorer les différentes activités d'enseignement visant le développement de la compétence culturelle utilisées dans la formation des infirmières.

\section{Recension des écrits}

Une combinaison des mots clés cultural competence, transcultural nursing, cultural safety et nursing education a été utilisée dans les bases de données CINAHL, Medline et Embase. La recherche a été limitée aux années 2008 à 2015 inclusivement, sauf en ce qui concerne les essais cliniques que nous avons intégré après en avoir fait la lecture dans Horvat et al. (2014). Les articles dans lesquels la culture était associée à l'ethnie uniquement ont été éliminés, afin de respecter la définition de la culture et celle de la compétence culturelle considérées dans le modèle du développement de la compétence culturelle (Blanchet Garneau, 2013). Huit publications ont été retenues. Malgré qu'elle cible l'ensemble des professionnels de la santé plutôt que les étudiantes en sciences infirmières précisément, une revue Cochrane (Horvat et al., 2014) a été retenue pour son niveau d'évidence élevé selon l'ordre hiérarchique établi par DiCenso, Guyatt, et Ciliska (2005). Une revue systématique (Allen, 2010) a aussi été conservée pour la même raison. Ensuite, une revue narrative (Long, 2012) et cinq études primaires (Allen, Brown, Duff, Nesbitt, et Hepner, 2013; Aponte, 2012; Cuellar, Brennan, Vito, et de Leon Siantz, 2008; Hawala-Druy et Hill, 2012; Munoz, DoBroka, et Mohammad, 2009) ont été retenues parmi plus de 60 articles pour la rigueur et la précision dans leur description d'une activité d'enseignement mise à l'essai avec des étudiantes au premier cycle en sciences infirmières. L'analyse des écrits est présentée selon les quatre thèmes suivants : les stratégies d'intégration, les activités d'enseignement, la réflexion sur soi et l'apprentissage dans l'action.

\section{Stratégie d'intégration}

Les stratégies pour intégrer les activités visant le développement de la compétence culturelle dans un programme de sciences infirmières diffèrent d'une université à l'autre. Pour 
certaines, ces activités sont condensées dans un seul cours (Allen et al., 2013; Aponte, 2012; Hawala-Druy et Hill, 2012; Munoz et al., 2009). La durée des cours décrits dans les études citées précédemment variait de deux à quatre heures par semaine pour une période de 7 à 15 semaines. Pour d'autres, elles sont intégrées dans différents cours tout au long du programme (Cuellar et al., 2008). L'état actuel des connaissances ne permet pas d'identifier une meilleure stratégie d'intégration de la compétence cultuelle dans un programme. Afin de faire un choix, il est important de considérer les besoins du milieu académique, les ressources disponibles, la position des enseignants et des personnes responsables du programme envers le projet ainsi que leur pouvoir décisionnel (Heagney, 2011; Project Management Institute, 2008).

\section{Activités d'enseignement}

À partir d'une recherche rigoureuse, Horvat, Horey, Romios, et Kis-Rigo (2014) ont repéré et analysé cinq essais cliniques randomisés ayant évalué les résultats d'une stratégie d'enseignement visant à développer les habiletés communicationnelles, la sensibilité culturelle ou la compétence culturelle des professionnels de la santé (Harmsen et al., 2005; Majumdar, Browne, Roberts, et Carpio, 2004; Sequist et al., 2010; Thom, Tirado, Woon, et McBride, 2006; Wade et Bernstein, 1991). La discussion en groupe est souvent la méthode choisie pour amener les intervenants à réfléchir sur leurs propres croyances et leurs valeurs et à prendre conscience de leurs préjugés (Harmsen et al., 2005; Thom et al., 2006; Wade et Bernstein, 1991). Nonobstant les activités d'enseignement analysée dans ces essais cliniques, les chercheurs ont remarqué des effets positifs sur les résultats de santé des patients (Majumdar et al., 2004; Sequist et al., 2010; Thom et al., 2006), sur leur taux de présence aux rendez-vous (Wade et Bernstein, 1991), sur leur implication dans les soins (Harmsen et al., 2005; Majumdar et al., 2004) ainsi que sur leur satisfaction des soins ou des services (Harmsen et al., 2005; Majumdar et al., 2004; Thom et al., 2006; Wade et Bernstein, 1991). La revue systématique d'Allen (2010), pour sa part, permet d'avoir un aperçu des principales activités d'enseignement utilisées aux États-Unis, en Europe, au Canada et en Australie. Treize études ayant évalué de manière qualitative ou quantitative les résultats d'une activité d'enseignement mise à l'essai auprès d'étudiantes en sciences infirmières ont été retenues pour cette revue. Les activités évaluées dans ces articles sont nommées, mais ne sont pas détaillées. L'enseignement magistral, la discussion, la simulation, le jeu de rôle, la consultation avec des experts de la compétence culturelle et le cours en ligne en sont quelques exemples. La majorité des activités d'enseignement présentées dans les études analysées par Allen (2010) ont permis un apprentissage de l'ordre de la compréhension de la complexité des soins en contexte de diversité culturelle, des habiletés culturelles, de la sensibilité culturelle ou des connaissances culturelles. À partir de ses résultats, Allen (2010) recommande d'amener les étudiantes à comprendre les facteurs sociopolitiques qui influencent la relation entre l'infirmière et le patient et ceux qui contribuent aux situations d'inégalités dans les soins. Toutefois, Allen ne donne pas de précision sur ces facteurs ni sur la manière d'accompagner les étudiantes dans leur apprentissage. À partir de l'étude de Hagey et MacKay (2000), Allen admet que l'enseignement de connaissances sur différents groupes culturels n'est pas suffisant pour réduire les attitudes racistes des étudiantes.

\section{Réflexion sur soi}

Les étudiantes sont souvent amenées à réfléchir sur leurs croyances et leurs valeurs durant les activités d'enseignement visant le développement de leur compétence culturelle (Allen et al., 2013; Aponte, 2012; Cuellar et al., 2008; Hawala-Druy et Hill, 2012; Munoz et al., 2009). Par exemple, dans le cours présenté par Aponte (2012), les étudiantes étaient invitées à partager 
leurs croyances en lien avec différents sujets tels que la grossesse, la santé mentale et la mort afin qu'elles prennent conscience de la diversité culturelle. Des questions de discussion précises leur étaient posées sur un forum en ligne pour amorcer la discussion. Toutefois, la manière dont les étudiantes étaient accompagnées durant leur réflexion n'est pas spécifiée dans l'article. Après avoir suivi ce cours, les étudiantes se disaient plus conscientes de l'importance de développer leur compétence culturelle et plus prêtes à offrir des soins culturellement adaptés (Aponte, 2012).

\section{Apprentissage dans l'action}

Parmi les activités d'enseignement visant le développement de la compétence culturelle d'étudiantes en sciences infirmières, le stage en milieu communautaire est utilisé dans plusieurs programmes afin de favoriser l'apprentissage à partir d'expériences en contexte de diversité culturelle (Aponte, 2012; Hawala-Druy et Hill, 2012; Long, 2012). Allen et al. (2013) considèrent aussi la simulation clinique, le jeu de rôle et la discussion à partir d'une situation infirmière clinique comme des expériences permettant aux étudiantes de saisir la complexité des soins culturellement compétents. L'apprentissage dans l'action a aussi été utilisé dans le cours de sept semaines présenté par Munoz et al. (2009). Ce cours multidisciplinaire a été mis à l'essai auprès d'étudiantes en sciences infirmières, en éducation et en travail social d'une université américaine. Selon Munoz et al. (2009), l'interaction entre des étudiantes provenant de milieux diversifiés favorise le développement de leur sensibilité, de leurs connaissances et de leurs habiletés culturelles pour travailler avec une population de plus en plus diversifiée. Les étudiantes étaient invitées à critiquer et à questionner ce qu'elles avaient entendu, lu ou expérimenté durant leur stage et à partager leur réflexion avec leurs collègues. Le racisme, l'homophobie, l'égalité d'accès, les différentes classes sociales et la justice sociale faisaient partie des sujets soulevés par les étudiantes (Munoz et al., 2009). Les auteurs ne précisent pas la manière dont les enseignants accompagnaient les étudiantes durant la discussion.

\section{Synthèse de la recension des écrits}

La variété d'activités d'enseignement présentée dans les études recensées ne permet pas d'en identifier une plus efficace que les autres pour stimuler le développement de la compétence culturelle des infirmières. Par contre, les chercheurs sont optimistes quant au potentiel d'améliorer la qualité des soins en développant la compétence culturelle des professionnels de la santé. On remarque que la réflexion est au cœur de ces activités d'enseignement qui prennent diverses formes telles que des discussions en groupe, des simulations ou des jeux de rôle. Par ailleurs, l'interaction entre les étudiantes leur permet de partager leurs croyances et leurs opinions et de stimuler leur réflexion sur la diversité culturelle dans les soins. Ainsi, une activité réflexive en groupe semble être une avenue prometteuse pour stimuler le développement de cette compétence chez des étudiantes en sciences infirmières.

\section{Élaboration de l'activité d'enseignement}

Selon une vision constructiviste de la culture et de la compétence culturelle (Blanchet Garneau, 2013) et à partir des résultats de la recension des écrits présentés ci-haut, une activité permettant aux étudiantes de réfléchir sur une expérience de soins vécue avec une personne/famille ayant différentes croyances ou valeurs qu'elles en lien avec leur état de santé a été élaborée. L'activité réflexive a été élaborée pour des étudiantes d'un programme québécois (Canada) de baccalauréat en sciences infirmières. Ce programme de formation initiale est d'une durée de trois ans. Il est construit selon une approche par compétences, mais la compétence culturelle n'est pas explicitement nommée dans le référentiel de compétences. Les étudiantes 
vivent des expériences de stage dès la première année du programme et elles ont alors l'opportunité de vivre des rencontres culturelles. Par contre, à notre connaissance, il n'y a pas d'activité réflexive dans le programme leur permettant de discuter en groupe de leurs expériences de soins en contexte de diversité culturelle. Afin de préciser la manière dont la réflexion des étudiantes sur la pratique infirmière en contexte de diversité culturelle pouvait être stimulée, des articles clés portant sur la pratique réflexive en sciences infirmières ont été consultés. Des écrits de Kolb (1984) et de Johns (2010) ont été retenus afin d'élaborer des questions de réflexion. Les quatre coauteures ont travaillé en collaboration afin de préciser les modalités de l'activité.

\section{Pratique réflexive}

Selon Gustafsson, Asp, et Fagerberg (2007), les concepts de réflexivité, de pratique réflexive, de pensée critique et de processus réflexif sont utilisés comme des synonymes dans la littérature scientifique. Malgré l'absence de consensus quant à la définition de la pratique réflexive (Atkins et Murphy, 1993), des auteurs s'entendent pour dire qu'elle permet de soutenir l'analyse critique des connaissances et de l'expérience afin de favoriser la compréhension d'une situation donnée (Mann, Gordon, et MacLeod, 2009). La pratique réflexive est, la plupart du temps, déclenchée par un événement qui surprend le praticien (Atkins et Murphy, 1993; Mann et al., 2009). Contrairement à toute réflexion spontanée, la pratique réflexive permet d'analyser une situation donnée sous différents angles de manière structurée et encadrée (Schön, 1983). La réflexion sur une situation vécue, aussi nommée réflexion sur l'action (Schön, 1983), peut se faire de manière individuelle ou en petit groupe (Mann et al., 2009). Étant donné l'importance du processus de réflexion et d'action dans le développement de la compétence culturelle des infirmières (Blanchet Garneau, 2013), il est pertinent d'utiliser un modèle permettant de stimuler la réflexion des étudiantes sur leur pratique en contexte de diversité culturelle. Le modèle de l'apprentissage expérientiel de Kolb (1984) a été retenu pour structurer l'activité réflexive. Selon Kolb (1984), l'apprentissage expérientiel permet de considérer l'apprentissage comme un processus influencé par l'expérience de l'étudiante, qu'elle soit personnelle ou en lien avec ses études. Selon ce chercheur, un apprentissage se fait lorsque des savoirs sont créés ou transformés de manière consciente, à partir d'une expérience. Élaboré à partir des travaux de Dewey, Lewin et Piaget, le cycle de l'apprentissage expérientiel de Kolb (1984) permet à l'étudiant de décrire une expérience (expérience concrète), de l'analyser (observation réflexive), d'amener une nouvelle perspective à la situation de départ (conceptualisation abstraite) et de transférer les apprentissages réalisés dans la pratique (expérimentation active). En d'autres mots, le cycle de Kolb permet de générer une nouvelle compréhension d'une situation vécue, d'intégrer les apprentissages réalisés grâce à la réflexion dans de nouvelles expériences semblables et de poursuivre la réflexion sur ces nouvelles expériences. Par contre, Kolb ne propose pas de questions de réflexion pour chacune des phases du cycle. Ainsi, afin d'opérationnaliser l'activité réflexive, celles du modèle de réflexion structurée de Johns (2010) ont été intégrées dans chacune des phases du cycle de Kolb (1984). Les questions de Johns pouvant s'intégrer dans les phases du cycle de Kolb (1984) ont été sélectionnées en collaboration avec la personne-ressource du projet, une coauteure. Cette personne était alors titulaire d'un cours de deuxième cycle portant sur la pratique réflexive en sciences infirmières. Le modèle de réflexion combinant les étapes de Kolb et les questions de Johns élaboré pour guider l'activité réflexive est illustré à la figure 1. 
Figure 1: Représentation schématique de l'intégration de questions de réflexion inspirées de Johns (2010) dans le cycle de l'apprentissage expérientiel de Kolb (1984)

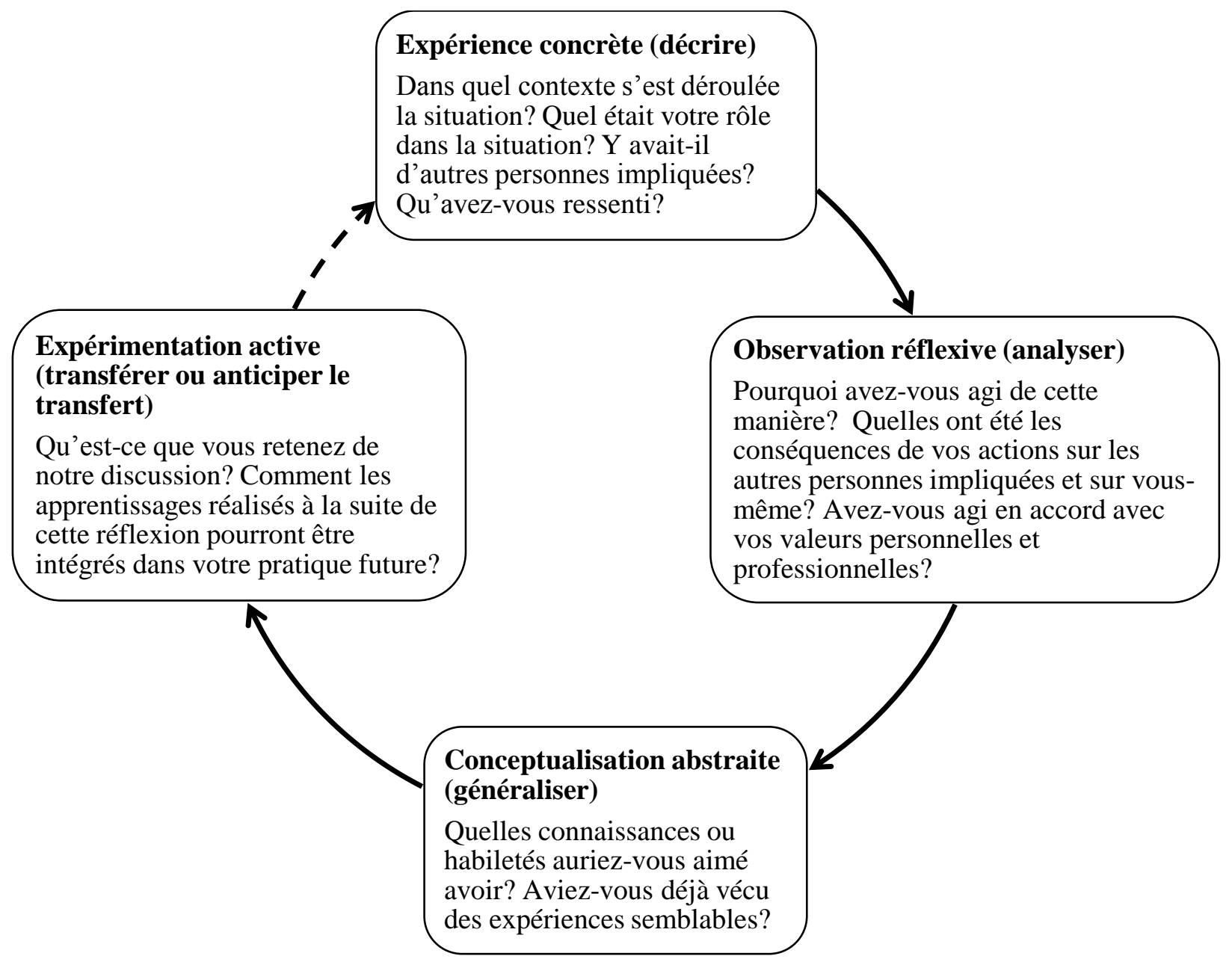

Selon la vision de Kolb (1984), le processus de réflexion permet à l'étudiant de générer de nouvelles connaissances à partir d'expériences antérieures afin de les transférer dans de nouvelles situations. Selon cette optique, l'activité devrait idéalement être réalisée à plusieurs reprises avec les mêmes étudiants afin de boucler le cycle de l'apprentissage expérientiel. Si le contexte ne permet pas de refaire l'activité, les étudiantes pourraient discuter de la manière dont elles pourraient transférer l'apprentissage réalisé durant l'activité dans leur pratique clinique. C'est pourquoi la flèche entre l'expérimentation active et l'expérimentation concrète est en pointillé à la figure 1 .

\section{Structure de l'activité}

L'activité réflexive a été élaborée pour un petit groupe d'étudiantes en sciences infirmières et pour une durée de 90 minutes, correspondant à leur disponibilité. Le rôle de l'animatrice consistait à introduire l'activité et de stimuler la réflexion des étudiantes durant la discussion. Les étudiantes, pour leur part, devaient se remémorer une situation vécue en stage 
avec une personne qui avait des valeurs ou des croyances qu'elles percevaient différentes des leurs. Les souvenirs qu'elles en gardaient pouvaient être positifs ou négatifs. Les consignes de départ étaient volontairement générales pour éviter de guider les étudiantes vers un type de situation précis. Chaque étape de l'activité réflexive est décrite et justifiée dans le tableau 1. La discussion en groupe constitue la partie centrale de l'activité réflexive. Pour chaque situation présentée, l'animatrice guide les étudiantes à travers les étapes du cycle de Kolb à l'aide des questions adaptées du modèle de réflexion structurée de Johns (figure 1).

Tableau 1 : Déroulement de l'activité réflexive

\begin{tabular}{|c|c|c|}
\hline $\begin{array}{l}\text { Étapes } \\
\text { (temps) }\end{array}$ & Description & Justification \\
\hline $\begin{array}{l}\text { Introduction } \\
(10 \mathrm{~min})\end{array}$ & $\begin{array}{l}\text { L'animatrice introduit l'activité et en } \\
\text { présente le déroulement. }\end{array}$ & $\begin{array}{l}\text { Établir un climat de confiance afin que } \\
\text { les participantes soient à l'aise } \\
\text { d'exprimer leur opinion (Martineau et } \\
\text { Simard, 2001); } \\
\text { Clarifier les concepts centraux de la } \\
\text { discussion pour guider le choix des } \\
\text { situations (Belknap, 2008). }\end{array}$ \\
\hline $\begin{array}{l}\text { Partie } \\
\text { individuelle } \\
\text { ou en dyade } \\
(5 \text { min })\end{array}$ & $\begin{array}{l}\text { Les participantes préparent la } \\
\text { présentation d'une situation au reste } \\
\text { du groupe. Elles écrivent les idées qui } \\
\text { leur viennent spontanément en lien } \\
\text { avec leur situation et elles utilisent les } \\
\text { questions de la boîte expérience } \\
\text { concrète du modèle de réflexion } \\
\text { (figure 1) pour se remémorer la } \\
\text { situation vécue. }\end{array}$ & $\begin{array}{l}\text { Clarifier leur perception de la situation } \\
\text { vécue ainsi que les émotions soulevées } \\
\text { par celle-ci afin d'avoir un point de } \\
\text { départ dans leur réflexion (Johns, } \\
\text { 2010). }\end{array}$ \\
\hline $\begin{array}{l}\text { Discussion } \\
\text { en groupe } \\
\text { (1h) }\end{array}$ & $\begin{array}{l}\text { Chacune leur tour, les participantes } \\
\text { présentent une situation vécue à leurs } \\
\text { collègues. Les étudiantes discutent de } \\
\text { chaque situation. L'animatrice guide la } \\
\text { discussion à partir des questions de } \\
\text { réflexion (figure 1). }\end{array}$ & $\begin{array}{l}\text { Stimuler la réflexion des étudiantes sur } \\
\text { leurs valeurs et leurs croyances ainsi } \\
\text { que sur leur pratique en contexte de } \\
\text { diversité culturelle afin de favoriser } \\
\text { des soins culturellement compétents } \\
\text { (Allen, 2010; Aponte, 2012; Cuellar et } \\
\text { al., 2008; Munoz et al., 2009); } \\
\text { Permettre à l'étudiante de préciser son } \\
\text { point de vue par rapport à une } \\
\text { situation et de le mettre en question à } \\
\text { partir de l'interaction avec les autres } \\
\text { participantes (Martineau et Simard, } \\
\text { 2001). }\end{array}$ \\
\hline $\begin{array}{l}\text { Complétion } \\
\text { du } \\
\text { questionnaire } \\
\text { (15 min) }\end{array}$ & $\begin{array}{l}\text { Les participantes remplissent de } \\
\text { manière anonyme le questionnaire. }\end{array}$ & $\begin{array}{l}\text { Évaluer les retombées de l'activité } \\
\text { réflexive afin d'émettre des } \\
\text { recommandations et d'améliorer } \\
\text { l'activité dans le futur. }\end{array}$ \\
\hline
\end{tabular}


Un questionnaire d'évaluation de l'activité a aussi été élaboré afin de connaître la perception des étudiantes sur l'apprentissage réalisé ainsi que sur leur appréciation générale de l'activité. Les 15 dernières minutes de l'activité étaient réservées pour que les étudiantes puissent le compléter, de manière anonyme. Les réponses des participantes à ce questionnaire sont présentées plus loin.

\section{Mise à l'essai de l'activité réflexive}

Une fois l'approbation obtenue du comité d'éthique de la recherche en santé de l'université où s'est déroulé le stage, toutes les étudiantes inscrites à l'hiver 2015 au cours de santé communautaire du programme ciblé pour mettre l'activité à l'essai ont été invitées à participer à l'activité. Elles ont d'abord reçu l'information nécessaire de manière écrite via leur forum de cours. Par la suite, le projet leur a été présenté de manière plus détaillée en classe, sous forme d'une présentation magistrale d'une durée de 30 minutes. Parmi ces étudiantes, 12 se sont portées volontaires. Comme elles n'étaient pas toutes disponibles au même moment, elles ont été séparées en deux groupes de cinq étudiantes et un groupe de deux étudiantes. Ce cours a été choisi comme porte d'entrée pour le recrutement des participantes parce que les étudiantes avaient déjà rencontré des situations cliniques, ce qui était nécessaire pour réaliser l'activité. Elles étaient d'ailleurs en stage en milieu communautaire au moment de mettre l'activité réflexive à l'essai. Il y avait aussi une ouverture de la part des responsables du cours pour l'activité. La première auteure a joué le rôle de l'animatrice pour les trois séances.

\section{Participantes}

Les participantes se situaient à la $2^{\mathrm{e}}$ session de la $2^{\mathrm{e}}$ année $(\mathrm{n}=2)$ ou à la $1^{\mathrm{e}}$ session de la $3^{\mathrm{e}}$ année $(n=10)$ du programme. Elles avaient des cheminements personnels variés. Quatre personnes étaient nées et avaient grandi en Amérique du Nord. Les huit autres étaient nées dans les Caraïbes, en Afrique, en Europe ou en Asie. Parmi celles-ci, quatre personnes vivaient en Amérique du Nord depuis moins de six ans. La moitié des participantes avaient une expérience de travail comme préposée aux bénéficiaires ou comme externe en soins infirmiers.

\section{Ajustement à l'activité}

Les constats réalisés à la suite de la première mise à l'essai de l'activité ont permis de faire des ajustements avec le deuxième et le troisième groupe. Pour pallier au manque de temps, les étudiantes du deuxième groupe ont été invitées à se placer en dyade dès la deuxième étape de l'activité (tableau 1). De plus, plutôt que de poser systématiquement toutes les questions de réflexion, les plus pertinentes ont été ciblées par l'animatrice au fur et à mesure de la discussion, tout en respectant les quatre phases de Kolb (1984).

\section{Situations discutées}

Chaque participante a présenté une situation dans laquelle elle percevait un écart quant aux croyances entre elle ou une autre infirmière et un patient/ famille. Les situations cliniques décrites par les étudiantes s'étaient principalement déroulées durant un stage, en santé communautaire, à l'urgence ou en périnatalité. Une étudiante a présenté une situation dans laquelle sa préceptrice avait des croyances différentes d'une patiente d'origine française quant à l'allaitement. Une autre étudiante a dit avoir été surprise des croyances d'une famille chinoise en lien avec les soins d'un nouveau-né. Deux étudiantes ont exposé une situation dans laquelle il y avait une barrière de langue entre l'infirmière et le patient. Une des situations discutées portait sur une expérience de discrimination vécue par une stagiaire d'origine africaine se disant non 
québécoise en contexte de soins à domicile. Les situations racontées par les participantes n'impliquaient pas toutes une différence en lien avec le pays d'origine. À titre d'exemple, une des participantes a décrit une situation vécue lors d'un stage à la salle d'accouchement. Elle devait s'occuper d'une patiente qui avait trois enfants de trois pères différents et qui avait eu recours à l'insémination pour en avoir un quatrième. En analysant la situation, l'étudiante a dit que son approche avec la patiente aurait pu être influencée par ses préjugés, mais que l'attitude des membres de l'équipe de soins lui a fait réaliser que son rôle était de prendre soin de cette femme, peu importe ses propres valeurs. Les questions d'analyse ont permis aux participantes de poser des hypothèses sur ce qui influençait l'attitude des infirmières de cette équipe. Selon les participantes, l'assistante infirmière-chef ou les infirmières leaders de l'équipe ont un grand rôle à jouer sur l'attitude des autres membres de l'équipe. Certaines ont mentionné de façon générale que les valeurs prônées par l'hôpital pourraient influencer les pratiques directes aux patients : « Ça se peut que ça soit l'esprit de l'hôpital qui ait un impact sur les différents leaders de chaque unité » $\left(\mathrm{G} 2^{2}\right)$. Les participantes n'ont pas précisé les valeurs en question, mais il est possible de penser que, par exemple, la position d'un établissement de santé par rapport à la participation des familles aux soins, au ratio infirmière-patient, aux valeurs de respect, d'ouverture à l'autre, d'humanisme puissent influencer les pratiques. Les questions de la phase conceptualisation abstraite ont amené une discussion sur les stratégies permettant d'éviter aux infirmières d'être influencées par leurs préjugées dans leurs soins. Les participantes ont soulevé la pertinence de prendre conscience de leurs préjugés et de connaître leurs limites personnelles et professionnelles par rapport aux demandes des patients, ce qu'elles sont prêtes à accepter. Elles ont discuté de l'importance d'en parler aux autres membres de l'équipe si elles ressentent un malaise envers un patient. En leur demandant ce qu'elles retenaient de la discussion (anticiper le transfert), les étudiantes ont nommé l'influence des pairs sur leur approche de soins. Cette situation a favorisé une discussion sur l'écart entre l'infirmière et le patient quant aux valeurs personnelles ainsi que sur l'influence de collègues plus expérimentées, qu'elles souhaitent imiter pour le respect qu'elles démontrent envers les personnes soignées. Ces deux éléments se retrouvent dans le premier niveau du développement de la compétence culturelle de Blanchet Garneau (2013), illustrés au tableau 2.

\section{Retombées de la mise à l'essai}

Les retombées de la mise à l'essai ont été examinées sous l'angle du développement de la compétence culturelle. L'analyse a été faite à partir des enregistrements audio des groupes de discussion et des réponses des étudiants au questionnaire d'évaluation de l'activité.

\section{Lien entre l'activité et le développement de la compétence culturelle}

L'analyse des propos des participantes lors des discussions en groupe a permis de faire le lien entre les idées véhiculées par celles-ci durant les discussions et les éléments de la compétence culturelle, tels que développés par Blanchet Garneau. Le tableau 2 présente l'évolution des trois niveaux de développement (Blanchet Garneau et Pepin, 2015b). Les éléments discutés par les étudiantes durant l'activité réflexive ont été mis en italique dans le tableau. La majorité des éléments du premier niveau ont été abordés. L'activité réflexive a aussi favorisé la discussion d'éléments du deuxième niveau de la composante construire la relation avec l'autre.

\footnotetext{
${ }^{2} \mathrm{G} 2$ : étudiante du 2e groupe
} 
Tableau 2 : Thèmes abordés lors des discussions en lien avec les trois niveaux du développement de la compétence culturelle [traduction par Blanchet Garneau] (Blanchet Garneau et Pepin, 2015, p. 1066)

\begin{tabular}{|c|c|c|}
\hline $\begin{array}{l}\text { Niveau 1- S'ouvrir aux } \\
\text { différentes réalités entourant } \\
\text { la pratique en contexte de } \\
\text { diversité culturelle }\end{array}$ & $\begin{array}{l}\text { Niveau } 2 \text { - Mettre à } \\
\text { l'épreuve sa pratique en } \\
\text { contexte de diversité } \\
\text { culturelle }\end{array}$ & $\begin{array}{l}\text { Niveau } 3 \text { - Réunir les } \\
\text { différentes réalités de la } \\
\text { pratique en contexte de } \\
\text { diversité culturelle }\end{array}$ \\
\hline $\begin{array}{l}\text { Construire la relation avec } \\
\text { l'autre } \\
\text { - Perçoit un écart entre } \\
\text { l'infirmière et le patient } \\
\text { - Démontre de l'ouverture } \\
\text { - Prend conscience de } \\
\text { l'importance } \\
\text { de la communication } \\
\text { Sortir du cadre habituel de } \\
\text { pratique } \\
\text { - Prend conscience des défis } \\
\text { entourant les soins en contexte } \\
\text { de diversité culturelle } \\
\text { - Prend conscience des limites } \\
\text { structurelles } \\
\text { Réinventer sa pratique dans } \\
\text { l'action } \\
\text { - Prend conscience de l'écart } \\
\text { entre la théorie et la pratique } \\
\text { - Imite l'approche de collègues } \\
\text { plus expérimentés }\end{array}$ & $\begin{array}{l}\text { Construire la relation avec } \\
\text { l'autre } \\
\text { - Découvre des points } \\
\text { communs entre l'infirmière } \\
\text { et le patient } \\
\text { - Se centre sur le patient } \\
\text { - Recourt à une variété de } \\
\text { stratégies de } \\
\text { communication } \\
\text { Sortir du cadre habituel } \\
\text { de pratique } \\
\text { - Prend en considération } \\
\text { l'environnement du patient } \\
\text { dans ses soins } \\
\text { - Compose avec les limites } \\
\text { structurelles } \\
\text { Réinventer sa pratique } \\
\text { dans l'action } \\
\text { - Prendre le risque de } \\
\text { changer } \\
\text { - Crée son modèle de } \\
\text { pratique }\end{array}$ & $\begin{array}{l}\text { Construire la relation avec } \\
\text { l'autre } \\
\text { - Intègre la différence dans sa } \\
\text { relation avec le patient } \\
\text { - Établit un partenariat avec le } \\
\text { patient } \\
\text { - Communique avec } \\
\text { discernement } \\
\text { Sortir du cadre habituel de } \\
\text { pratique } \\
\text { - Considère le patient et } \\
\text { l'infirmière comme partie } \\
\text { intégrante d'un système } \\
\text { dynamique } \\
\text { - Déjoue les limites } \\
\text { structurelles en s'engageant } \\
\text { dans son développement } \\
\text { professionnel } \\
\text { Réinventer sa pratique dans } \\
\text { l'action } \\
\text { - Agit au bon moment } \\
\text { - Intègre son modèle dans sa } \\
\text { pratique }\end{array}$ \\
\hline
\end{tabular}

Il est possible d'observer une tendance entre les éléments de la compétence culturelle (tableau 2) abordés durant la discussion et les questions des quatre phases réflexives (figure 1). Tout d'abord, lorsque les participantes décrivaient leurs expériences, elles avaient tendance à parler de la construction de la relation avec l'autre. Quelques étudiantes ont démontré de l'ouverture en spécifiant qu'elles avaient pris conscience de leurs préjugés envers un patient. L'étudiante dont la situation est présentée au paragraphe précédent a même dit être passée pardessus ses préjugés pour se centrer sur les besoins de la patiente. Cet élément se trouve au deuxième niveau du modèle de Blanchet Garneau (2013). L'analyse des retombées de la réflexion en groupe ne permet pas de déterminer si la prise de conscience s'est faite avant l'activité ou grâce à celle-ci. Par contre, l'activité lui a permis de mettre des mots sur ses émotions en lien avec la situation vécue et de réaliser une pratique réflexive structurée avec ses collègues. 
En lien avec la composante prend conscience de l'importance de la communication, deux étudiantes ont indiqué avoir eu de la facilité à créer un lien avec un patient qui avait la même langue maternelle qu'elles. Une personne a dit avoir tenté de pallier à la barrière de langage en utilisant des gestes pour essayer d'expliquer à une patiente l'importance de garder son soluté. Elle a eu recourt à une variété de stratégies de communication, un élément du deuxième niveau (Blanchet Garneau, 2013).

Pour ce qui est des questions d'analyse et de généralisation, elles ont surtout amené les étudiantes à discuter des défis entourant les soins en contexte de diversité culturelle ainsi que des limites structurelles à l'adoption d'une approche prenant en considération la culture des personnes. Des participantes ont discuté du fait qu'elles avaient parfois de la difficulté à savoir comment intervenir avec certains patients, malgré les connaissances développées durant leur formation. En d'autres mots, elles faisaient le constat que leur programme actuel, sans tenir compte de l'activité mise à l'essai en tant que projet pilote, ne les préparait pas adéquatement à intervenir en contexte de diversité culturelle. Par exemple, le refus de patients de recevoir des soins de certaines infirmières en raison de leur apparence a été identifié comme une limite aux soins culturellement compétents. Dans cet extrait, deux participants se questionnent sur les motifs de refus d'un patient de recevoir des soins :

Étudiante 1 : Est-ce qu'il refuse tes soins parce que tu n'es pas de sa culture ou parce que tu es stagiaire?

Étudiante 2 : Je ne sais pas, je ne peux pas me prononcer parce qu'il a seulement dit 'un stagiaire non québécois'. Je ne sais pas s'il a refusé à cause de ça ou pas. $\left(\mathrm{G}^{3}{ }^{3}\right)$

Le manque de temps pour comprendre les besoins culturels de chaque personne a aussi été discuté dans les trois groupes. Durant la discussion, les étudiantes semblaient à l'aise d'exprimer leur désaccord envers leurs collègues tout en restant respectueuses et polies. Quelques étudiantes sont revenues sur leur position en entendant les arguments de leurs collègues.

Dans les trois groupes, les participantes ont indiqué qu'elles retenaient l'importance d'explorer les croyances des personnes en lien avec leur situation de soins. Dans le premier groupe, elles ont retenu l'importance de développer sa confiance en soi et d'être créatives afin de trouver la meilleure manière d'entrer en relation avec chaque patient. Dans le deuxième groupe, les étudiantes ont aussi retenu l'importance de s'inspirer de l'approche d'infirmières qui sont reconnues pour leur compétence culturelle tant par leurs pairs que par les patients..

\section{Perception des étudiantes de leur apprentissage}

La première section du questionnaire écrit comportait trois questions, élaborées à partir des trois dimensions du développement de la compétence culturelle selon Blanchet Garneau (2013) :

\section{Construire la relation avec l'autre.}

1. Durant cette activité, comment votre perception de la relation entre l'infirmière et le patient en contexte de diversité culturelle a-t-elle changé?

\section{Sortir du cadre habituel de pratique.}

\footnotetext{
${ }^{3} \mathrm{G} 1$ : étudiante du 1er groupe
} 
2. Qu'avez-vous appris sur les défis et les limites qui entourent la pratique infirmière en contexte de diversité culturelle?

\section{Réinventer sa pratique dans l'action.}

3. De quelle manière cette activité de réflexion pourrait-elle influencer votre pratique infirmière?

En lien avec leur perception de la relation entre l'infirmière et le patient, l'apprentissage réalisé par les étudiantes semble se situer au niveau de la prise de conscience. La majorité des étudiantes ont pris conscience de la différence entre l'infirmière et le patient, de la diversité culturelle parmi les patients, de l'impact de leurs croyances dans la qualité de leurs soins, de l'importance d'explorer les croyances du patient dans leur évaluation et du pouvoir de l'infirmière de diminuer les inégalités dans les soins de santé. Une étudiante a écrit : "Cette activité m'a vraiment fait réfléchir. Je vois maintenant que nos croyances et nos valeurs peuvent influencer les soins que l'on donne aux patients qui en ont des différentes. » $\left(\mathrm{G} 3^{4}\right)$.

En lien avec les défis et les limites, les étudiantes ont retenu différents facteurs pouvant nuire aux soins culturellement compétents, tant au niveau personnel qu'organisationnel. Au niveau personnel, les étudiantes ont noté l'influence des collègues sur les attitudes discriminatoires des infirmières. Une étudiante a noté qu'un des défis en tant que stagiaire ou nouveau membre d'une équipe de soins est d'adopter une approche sensible à la culture des patients tout en se faisant accepter dans l'équipe. Le manque de temps imposé par le contexte de soins actuel a été identifié par une étudiante comme une limite à l'approche de soins centrée sur le patient et sa culture. Selon quelques participantes, le manque de reconnaissance ou le manque de soutien de la part des infirmières-chefs pour des soins culturellement compétents pourrait limiter le développement de leur compétence culturelle. Une autre limite identifiée par une participante est le manque de préparation des infirmières à intervenir avec des personnes de différentes cultures : "Une des limites qu'on a en tant qu'infirmière est qu'on a à interagir avec des gens de toutes origines, mais que l'on n'aborde pas le sujet de la diversité culturelle à l'université »(G3).

Pour leur pratique infirmière future, les thèmes suivants sont ressortis de l'analyse des réponses des participantes au questionnaire: l'importance de démontrer de l'ouverture à comprendre l'autre, l'importance de prendre le temps de réfléchir sur leurs valeurs et sur leur pratique, l'importance de prévenir les situations d'injustice en reconnaissant la diversité culturelle dans les soins et l'importance d'offrir des soins égaux malgré les différences :

(...) je vais me rappeler qu'il est normal que certaines valeurs entrent en conflit, mais je vais me rappeler que la personne est comme elle est, et que malgré nos différences, je peux être caring et donner de bons soins (G3).

Selon les réponses des participantes, l'activité réflexive leur a permis de prendre conscience de l'impact que peut avoir l'écart entre l'infirmière et le patient sur la qualité des soins et de discuter des facteurs influençant la relation entre l'infirmière et le patient. On remarque aussi dans leurs réponses leur volonté de considérer la culture des patients dans leurs soins.

\footnotetext{
${ }^{4} \mathrm{G} 3$ : étudiante du groupe 3
} 
La deuxième section du questionnaire permettait de recueillir les commentaires généraux des étudiantes sur l'activité. La possibilité de pouvoir partager leurs expériences personnelles, la diversité culturelle parmi les participantes, la structure de la discussion ainsi que le nombre de participantes sont ressortis dans les éléments positifs de l'activité réflexive. De plus, la majorité des participantes ont trouvé que l'activité pouvait leur permettre d'améliorer leur pratique infirmière future. Le manque de temps a été le principal défi soulevé par les étudiantes. Les autres commentaires des étudiantes portaient essentiellement sur l'intégration de l'activité dans leur programme de Baccalauréat en sciences infirmières. Selon elles, la deuxième année est un bon moment pour aborder le sujet de la diversité culturelle dans les soins puisqu'elles ont alors déjà une meilleure idée de ce qu'implique la pratique infirmière. Une personne a noté la contribution que pourrait avoir une activité semblable durant les stages en santé mentale et en soins critiques puisque les étudiantes sont alors confrontées à « des patients parfois stigmatisés et où il y a des inégalités dans les soins » $\left(\mathrm{G} 2^{5}\right)$. Les participantes semblent avoir apprécié l'activité réflexive en général et elles auraient trouvé utile que toutes les étudiantes de leur cohorte y participent.

\section{Discussion}

L'accent de la discussion a été mis sur l'apprentissage des étudiantes, les forces et les limites de l'activité réflexive. Des recommandations sont aussi énoncées.

\section{Apprentissage}

L'activité réflexive guidée par des questions de réflexion inspirées de Johns (2010) et intégrées au cycle de l'apprentissage expérientiel de Kolb (1984) a permis, selon les étudiantes, de stimuler leur réflexion sur différentes réalités entourant la pratique en contexte de diversité culturelle, tel que suggéré par le modèle du développement de la compétence culturelle de Blanchet Garneau (2013). L'accent a été mis sur l'apprentissage à partir d'un processus de réflexion structurée ainsi que d'échange entre les participantes sur des situations proposées par ces dernières.

Même si la durée de l'activité réflexive était plus courte (90 min.) que celle des activités d'enseignement présentées dans les études sur le développement de la compétence culturelle des infirmières, les retombées sont comparables. En effet, les cours de Munoz (2009), Aponte (2012) et Hawala-Druy et Hill (2012), ayant inspiré l'élaboration de l'activité réflexive, ont aussi permis un apprentissage de l'ordre de la prise de conscience chez les étudiantes. Par exemple, les étudiantes ayant participé au cours de 14 heures présenté par Munoz (2009) se sont dites plus conscientes de la diversité ethnique et sociale dans la population. Ce cours permettait également aux étudiantes de discuter en elles et de partager leurs expériences culturelles vécues en stage. Comme les étudiantes avaient des journées de stage entre les cours, elles pouvaient intégrer rapidement leur apprentissage en milieu clinique. D'ailleurs, les enseignants ont noté une amélioration de l'efficacité des étudiantes à interagir avec une population diversifiée au fil des sept semaines du cours (Munoz, 2009). Le cours présenté par Aponte (2012) a aussi permis une prise de conscience chez les étudiantes de l'importance d'adopter une approche de soins centrée sur le patient et sa culture afin d'améliorer la qualité des soins. Dans ce cours d'une durée de 15 semaines, les étudiantes partageaient leurs réflexions avec leurs collègues de manière écrite, en ligne, et en personne, en classe. Cette formule leur a permis de réfléchir sur l'influence de leurs

\footnotetext{
${ }^{5} \mathrm{G} 2$ : Étudiante du groupe 2
} 
croyances et de leurs préjugés dans leurs relations avec des patients. Le cours présenté par Hawala-Druy et Hill (2012), encourageait aussi les étudiantes à discuter de la diversité culturelle à partir de thèmes précis. Des mises en situation et des lectures en lien avec les inégalités dans les soins, les populations vulnérables ou les facteurs influençant une relation de soins permettaient d'initier la discussion ou les débats. À la fin de ce cours de 14 semaines, les étudiantes devaient réfléchir sur l'évolution de leur compétence culturelle et de celle de leurs collègues durant le cours. Elles ont toutes exprimé l'importance de prendre conscience de leurs croyances et de leurs préjugés afin d'éviter que ceux-ci nuisent à leurs relations soins.

Comparativement aux résultats des articles recensés dans le cadre de ce projet, les participantes de l'activité réflexive semblaient préoccupées par les barrières à l'adoption d'une approche de soins culturellement compétents telles que leurs manque d'expérience, le manque de temps imposé par le contexte de soins ou le manque de soutien de la part de leurs collègues pour des soins culturellement compétents. Elles reconnaissaient l'importance d'une approche centrée sur la personne et sa culture et démontraient une volonté de l'adopter, mais trouvaient peu de solutions aux défis et aux limites soulevés. Il est possible que les questions proposées (figure 1) et les interventions de l'animatrice durant l'activité aient guidé les participantes vers ce type de réflexion. En effet, le modèle utilisé ne permet peut-être pas de motiver les étudiantes à trouver des pistes de solutions en lien avec les barrières soulevées.

\section{Forces de l'activité}

La principale force réside dans l'utilisation du modèle du développement de la compétence culturelle de Blanchet Garneau en combinaison avec un modèle de réflexion inspiré de Johns (2010) et de Kolb (1984) pour élaborer et structurer l'activité réflexive. La définition de la culture prise en compte dans le modèle de Blanchet Garneau a guidé les participantes à décrire des situations cliniques variées, pour lesquelles les différences culturelles ne se situaient pas uniquement au niveau de l'ethnicité. Le modèle de réflexion a permis de structurer le processus de réflexion nécessaire au développement de la compétence culturelle des étudiantes à travers les trois niveaux, selon le modèle de Blanchet Garneau. L'interaction entre les étudiantes est une autre force de l'activité. En effet, la variété d'opinions vis-à-vis les sujets abordés a pu stimuler la réflexion des participantes.

\section{Limites de l'activité réflexive}

Certains facteurs ont pu limiter les retombées de l'activité. Premièrement, les participantes avaient toutes déjà un intérêt envers le développement de la compétence culturelle, puisqu'elles se sont portées volontaires. D'ailleurs, plus de la moitié (huit) d'entre elles avaient grandi à l'extérieur du pays. Nous pouvons supposer que les étudiantes ayant déjà vécu un choc culturel ou s'étant déjà senties en situation de minorité s'intéressent davantage au développement de la compétence culturelle des infirmières. Le fait que la même personne ait animé et analysé l'activité à partir de ses observations amène un deuxième biais. Afin de réduire l'effet de ce facteur, l'animatrice a confirmé ou infirmé ses impressions en réécoutant l'enregistrement audio des groupes de discussion quelques jours après la tenue de l'activité. De plus, les verbatim ont été retranscrits pour faciliter l'analyse des thématiques abordées. Un troisième facteur qui a pu limiter l'apprentissage des participantes est le délai entre les situations vécues et l'activité réflexive. Certaines situations avaient eu lieu trois mois plus tôt. Les souvenirs se modifient avec le temps et la réflexion sur sa pratique peut être moins significative. Finalement, le fait d'avoir 
mis l'activité à l'essai à une seule reprise avec chaque groupe a pu limiter le développement de la compétence culturelle des étudiantes et, par le fait même, les retombées de cette activité.

\section{Recommandations pour la formation}

Afin de permettre aux étudiantes de devenir familières avec le processus de réflexion proposé et ainsi y avoir recourt de manière autonome dans leur pratique future, il serait intéressant de réaliser l'activité à quelques reprises dans le programme de formation initiale (Lasnier, 2000). L'activité pourrait aussi être utilisée par les préceptrices durant les stages. Les étudiantes pourraient alors transférer directement leur apprentissage dans leur pratique. Cela permettrait de boucler le cycle de l'apprentissage expérientiel tel que présenté par Kolb (1984). Reprendre l'activité favoriserait aussi l'évolution à travers les niveaux du développement de la compétence culturelle (Blanchet Garneau, 2013). En réalisant l'activité peu de temps après ou pendant les stages, les étudiantes pourraient présenter une situation qu'elles auraient vécue récemment. Ceci pallierait à l'altération des souvenirs avec le temps. C'est d'ailleurs ce que font les étudiantes du cours présenté par Munoz et al. (2009). Chaque semaine, pour cinq semaines, ces étudiantes doivent faire un travail de réflexion sur un événement vécu durant la semaine. Pour s'ajuster à la contrainte de temps, il pourrait être demandé aux étudiantes de mettre par écrit leur réflexion sur une situation vécue à partir des questions proposées pour les quatre phases (figure 1), en guise de préparation.

\section{Recommandation pour la recherche}

De futurs projets de recherche pourraient porter sur le développement d'indicateurs précis pour évaluer la compétence culturelle des étudiantes à partir du modèle de Blanchet Garneau. Une grille d'évaluation outillerait les enseignants à situer les étudiantes dans le processus du développement de leur compétence culturelle sans avoir recours à l'auto-évaluation ou en combinaison avec celle-ci. Les résultats pourraient être utilisés pour donner une rétroaction aux étudiantes et pour ajuster l'activité d'enseignement.

\section{Recommandations pour la pratique}

Comme la compétence culturelle de l'infirmière continue à se développer après la fin de la formation initiale, l'intégration de l'activité réflexive dans les unités de soins pourrait favoriser son développement. Le défi se trouve dans le type de soutien que l'organisation pourrait offrir aux infirmières pour mener à bien ce type d'activité dans leurs milieux de soins. L'engagement de l'organisation est essentiel à la réalisation et à la survie à long terme de ce type d'activité de développement professionnel.

\section{Conclusion}

L'activité d'enseignement présentée dans ce document visait à stimuler la réflexion d'étudiantes en sciences infirmières sur les différentes réalités entourant la pratique en contexte de diversité culturelle. Le modèle du développement de la compétence culturelle de Blanchet Garneau (2013) combiné à des notions de pratique réflexive s'est avéré utile et efficace pour l'élaboration de l'activité réflexive. Les participantes ont réalisé un apprentissage se situant au niveau de la prise de conscience de l'ampleur du phénomène ainsi que de leur potentiel pour réduire les situations d'inégalité dans les soins. Les participantes ont apprécié l'activité et encouragent son intégration dans leur programme de baccalauréat en sciences infirmières. 
Finalement, la pratique réflexive en groupe semble être une avenue prometteuse pour stimuler le développement de la compétence culturelle des infirmières. Elle pourrait être intégrée à la fois en formation initiale et dans les milieux cliniques. 


\section{Références}

AACN. (2008). Cultural competency in baccalaureate nursing education. Repéré à http://www.aacn.nche.edu/education-resources/cultural-competency

AIIC. (2010). Énoncé de position : Encourager la compétence culturelle dans les soins infirmiers. Repéré à https://cna-aiic.ca/ /media/cna/page-content/pdffr/ps114_cultural_competence_2010_f.pdf?la=fr

Allen, J. (2010). Improving cross-cultural care and antiracism in nursing education: a literature review. Nurse Education Today, 30(4), 314-320. doi:10.1016/j.nedt.2009.08.007

Allen, J., Brown, L., Duff, C., Nesbitt, P., et Hepner, A. (2013). Development and evaluation of a teaching and learning approach in cross-cultural care and antidiscrimination in university nursing students. Nurse Education Today, 33(12), 1592-1598. doi:10.1016/j.nedt.2012.12.006

Aponte, J. (2012). Cultural competency in baccalaureate US nursing education: hybrid course. Holistic Nursing Practice, 26(5), 243-258. doi:10.1097/HNP.0b013e318263f2c9

Atkins, S., et Murphy, K. (1993). Reflection: a review of the literature. Journal of Advanced Nursing, 18, 1188-1192. http://dx.doi.org/10.1046/j.1365-2648.1993.18081188.x

Beach, M. C., Price, E. G., Gary, T. L., Robinson, K. A., Gozu, A., Palacio, A., . . Cooper, L. A. (2005). Cultural competence: a systematic review of health care provider educational interventions. Medical Care, 43(4), 356-373. http://dx.doi.org/10.1097/01.mlr.0000156861.58905.96

Belknap, R. A. (2008). Teaching social justice using a pedagogy of engagement. Nurse Educator, 33(1), 9-12. doi:10.1097/01.NNE.0000299499.24905.39

Blanchet Garneau, A. (2013). Proposition théorique constructiviste du développement de la compétence culturelle infirmière. (Thèse doctorale, Université de Montréal, Montréal). Repéré à https://papyrus.bib.umontreal.ca/xmlui/handle/1866/10354

Blanchet Garneau, A., et Pepin, J. (2015a). Cultural competence: A constructivist definition. Journal of Transcultural Nursing, 26, 9-15.

Blanchet Garneau, A., et Pepin, J. (2015b). A constructivist theoretical proposition of cultural competence development in nursing. Nurse Education Today, 35, 1062-1068. doi:10.1016/j.nedt.2015.05.019

Campinha-Bacote, J. (2007). The process of cultural competence in the delivery of healthcare services: A culturally competent model of care (5e éd.). Cincinnati, $\mathrm{OH}$ : Transcultural C.A.R.E. Associates.

Campinha-Bacote, J. (2011). Coming to know cultural competence: an evolutionary process. International Journal for Human Caring, 15(3), 42-48.

Cuellar, N. G., Brennan, A. M., Vito, K., et de Leon Siantz, M. L. (2008). Cultural competence in the undergraduate nursing curriculum. Journal of Professional Nursing, 24(3), 143149. doi:10.1016/j.profnurs.2008.01.004 
DiCenso, A., Guyatt, G., et Ciliska, D. (2005). Evidence-Based Nursing: A guide to clinical practice. Saint-Louis, MO : Elsevier Mosby.

Gallagher, R. W., et Polanin, J. R. (2015). A meta-analysis of educational interventions designed to enhance cultural competence in professional nurses and nursing students. Nurse Education Today, 35(2), 333-340. doi:10.1016/j.nedt.2014.10.021

Giger, J. N., et Davidhizar, R. E. (2012). Transcultural nursing: assessment and intervention. $\left(6^{\mathrm{e}}\right.$ éd.). St-Louis, MO : Mosby.

Gustafsson, C., Asp, M., et Fagerberg, I. (2007). Reflective practice in nursing care: embedded assumptions in qualitative studies. International Journal of Nursing Practice, 13(3), 151160. doi:10.1111/j.1440-172X.2007.00620.x

Hagey, R., et MacKay, R. (2000). Qualitative research to identify racialist discourse: towards equity in nursing curricula. International Journal of Nursing Studies, 37(1), 45-56. http://dx.doi.org/10.1016/s0020-7489(99)00051-6

Harmsen, H., Bernsen, R., Meeuwesen, L., Thomas, S., Dorrenboom, G., Pinto, D., et Bruijnzeels, M. (2005). The effect of educational intervention on intercultural communication: results of a randomised controlled trial. British Journal of General Practice, 55(514), 343-350.

Hawala-Druy, S., et Hill, M. H. (2012). Interdisciplinary: Cultural competency and culturally congruent education for millennials in health professions. Nurse Education Today, 32(7), 772-778. doi:10.1016/j.nedt.2012.05.002

Heagney, J. (2011). Fundamentals of Project Management. (4 éd.). New York, NY : AMACOM.

Horvat, L., Horey, D., Romios, P., et Kis-Rigo, J. (2014). Cultural competence education for health professionals. Cochrane Database of Systematic Reviews, 5, CD009405. doi:10.1002/14651858.CD009405.pub2

Johns, C. (2010). Guided Reflection: A narrative approach to advancing professional practice. ( $2^{\mathrm{e}}$ éd.). Chichester, Royaume-Unis: Blackwell.

Kolb, D. (1984). Experiential learning: experience as the source of learning and development. Englewood Cliffs, CA: Prentice Hall.

Lasnier, F. (2000). Réussir la formation par compétences. Montréal, QC : Guérin.

Long, T. B. (2012). Overview of teaching strategies for cultural competence in nursing students. Journal of Cultural Diversity, 19(3), 102-108.

Majumdar, B., Browne, G., Roberts, J. et Carpio, B. (2004). Effects of cultural sensitivity training on health care provider attitudes and patient outcomes. Journal of Nursing Scholarship, 36(2), 161-166. http://dx.doi.org/10.1111/j.1547-5069.2004.04029.x

Mann, K., Gordon, J., et MacLeod, A. (2009). Reflection and reflective practice in health professions education: a systematic review. Advances in Health Sciences Education Theory and Practice, 14(4), 595-621. doi:10.1007/s10459-007-9090-2 
Martineau, S., et Simard, D. (2001). Les groupes de discussion. Québec, QC : Presses de l'Université du Québec.

McGibbon, E., Etowa, J., et McPherson, C. (2008). Health-care access as a social determinant of health. Canadian Nurse, 104(7), 22-27.

Munoz, C. C., DoBroka, C. C., et Mohammad, S. (2009). Development of a multidisciplinary course in cultural competence for nursing and human service professions. Journal of Nursing Education, 48(9), 495-503. doi:10.3928/01484834-20090610-03

Newbold, K. B. (2005). Self-rated health within the Canadian immigrant population: risk and the healthy immigrant effect. Social Science and Medicine, 60(6), 1359-1370. doi:10.1016/j.socscimed.2004.06.048

Project Management Institute (2008). Guide du corpus des connaissances en management de projet. ( $4^{\mathrm{e}}$ éd.). Newtown Square, PA : Project Management Institute, Inc.

Purnell, L. (2002). The Purnell Model for Cultural Competence. Journal of Transcultural Nursing, 13(3), 193-196.

RNAO. (2007). Embracing Cultural Diversity in Health Care: Developing Cultural Competence.

Schön, D. A. (1983). The reflective practitioner, how professionals think in action. New York, NY : Basic Books.

Sequist, T. D., Fitzmaurice, G. M., Marshall, R., Shaykevich, S., Marston, A., Safran, D. G., et Ayanian, J. Z. (2010). Cultural competency training and performance reports to improve diabetes care for black patients: a cluster randomized, controlled trial. Annals of Internal Medicine, 152(1), 40-46. doi:10.7326/0003-4819-152-1-201001050-00009

Smedley, B. D., Stith, A. Y., et Nelson, A. R. (2003). Unequal treatment: confronting racial and ethnic disparities in health care. Washington, D.C. : National Academies.

Thom, D. H., Tirado, M. D., Woon, T. L., et McBride, M. R. (2006). Development and evaluation of a cultural competency training curriculum. BMC Medical Education, 6, 38. doi:10.1186/1472-6920-6-38

van Boekel, L. C., Brouwers, E. P., van Weeghel, J., et Garretsen, H. F. (2013). Stigma among health professionals towards patients with substance use disorders and its consequences for healthcare delivery: systematic review. Drug and Alcohol Dependence, 131(1-2), 2335. doi:10.1016/j.drugalcdep.2013.02.018

Wade, P., et Bernstein, B. L. (1991). Culture sensitivity training and counselor's race: effects on black female clients' perceptions and attrition. Journal of Counseling Psychology, 28(1), 9-15. http://dx.doi.org/10.1037/0022-0167.38.1.9 